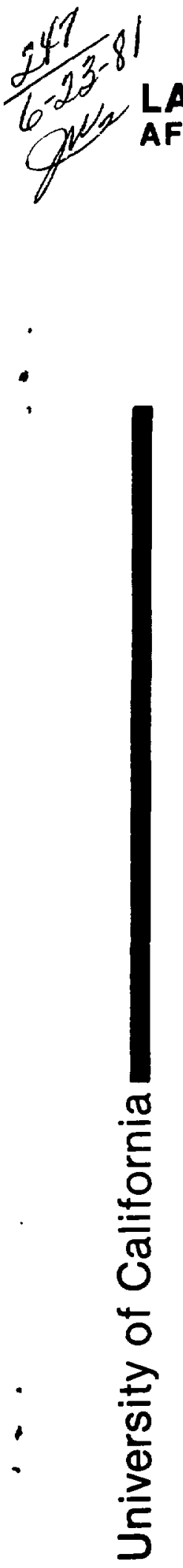

1

D. 2757

LA -8721

AFATL-TR-80-135

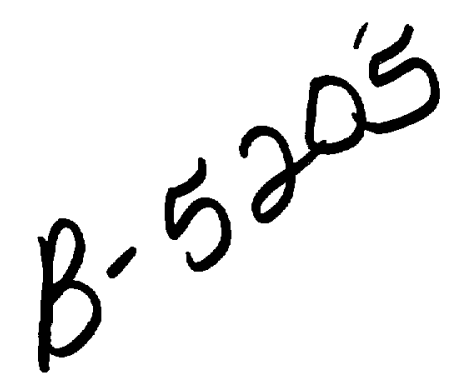

MASTER

Kriging Analysis of Uranium

Concentrations in Test Area C-74L,

Eglin Air Force Base, Florida

LOS ALAMOS SCIENTIFIC LABORATORY

Post Office Box 1663 Los Alamos. New Mexico 87545

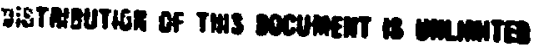




\title{
Kriging Analysis of Uranium Concentrations in Test Area C-74L, Eglin Air Force Base, Florida
}

\author{
Gary C. White
}

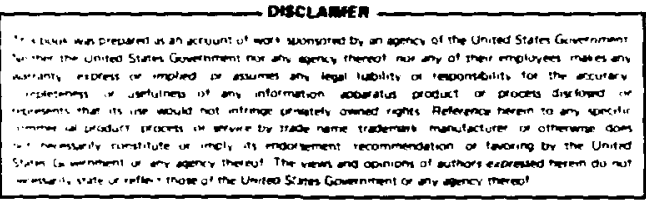




\title{
KRIGING ANALYSIS OF URANIUM CONCENTRATIONS IN TEST AREA C-74L, EGLIN AIR FORCE BASE, FLORIDA
}

by

Gary C. White

\begin{abstract}
Soil samples from Test Area C-74L, Eglin Air Force Base, Florida, were analyzed for depleted uranium by instrumental epithermal neutron activation analysis. The sampling design used was a modified polar coordinate scheme. The resulting data were analyzed with a statistical procedure called kriging to obtain a contour map of concentration and a 95\% confidence interval map. The majority of uranium remains in the center of the area near the target abutment.
\end{abstract}

\section{INTRODUCTION}

This report summarizes research of the Los Alamos National Laboratory for the Air Force Armament Laboratory, Eglin Air Force Base, Florida, from October 1, 1979 to September 30, 1980. This research emphasized sampling of Test Area (TA) C-74L to determine areas needing clean-up because of uranium contamination. Included are (1) the initial sampling design, (2) the analytical results, (3) an evaluation of the sampling design's effectiveness, and (4) an evaluation of kriging's ability to estimate uranium concentrations in the soil.

The objective of this study was to develop a procedure for evaluating decontamination requirements in TA C-74L. Kriging provides a method to determine areas needing clean-up (that is, areas where uranium concentrations exceed a predetermined level). This research has application in field situations where substantial amounts of uranium have been released to the environs and where an inventory to discover the fate of the contaminant is required.

\section{STUDY AREA}

TA C. $74 \mathrm{~L}$ is located in Okaloosa County, Florida, on Eglin Air Force Base in the Florida Panhandle. The portion of TA C-74L used for test firings consists of a cleared and level gun firing area $125 \times 46 \mathrm{~m}$. The soil, which is sand intermixed with clay, contains unburned propellants. metal fragments, and small pieces of plastic residue from gun firings. At the southeast end of the test area. the land slopes rapidly to a tributary of Rocky Creek. The distance from the slope top to the stream is approximately $75 \mathrm{~m}$.

\section{SAMPLING DESIGN}

The sampling design, shown in Fig. 1, was patterned after the recommendations of White et al. (1980), but modified slightly to make use of a grid already available at TA C-74L. The design was on a polar coordinate system with 12 concentric circles, each with a radius 7.6 


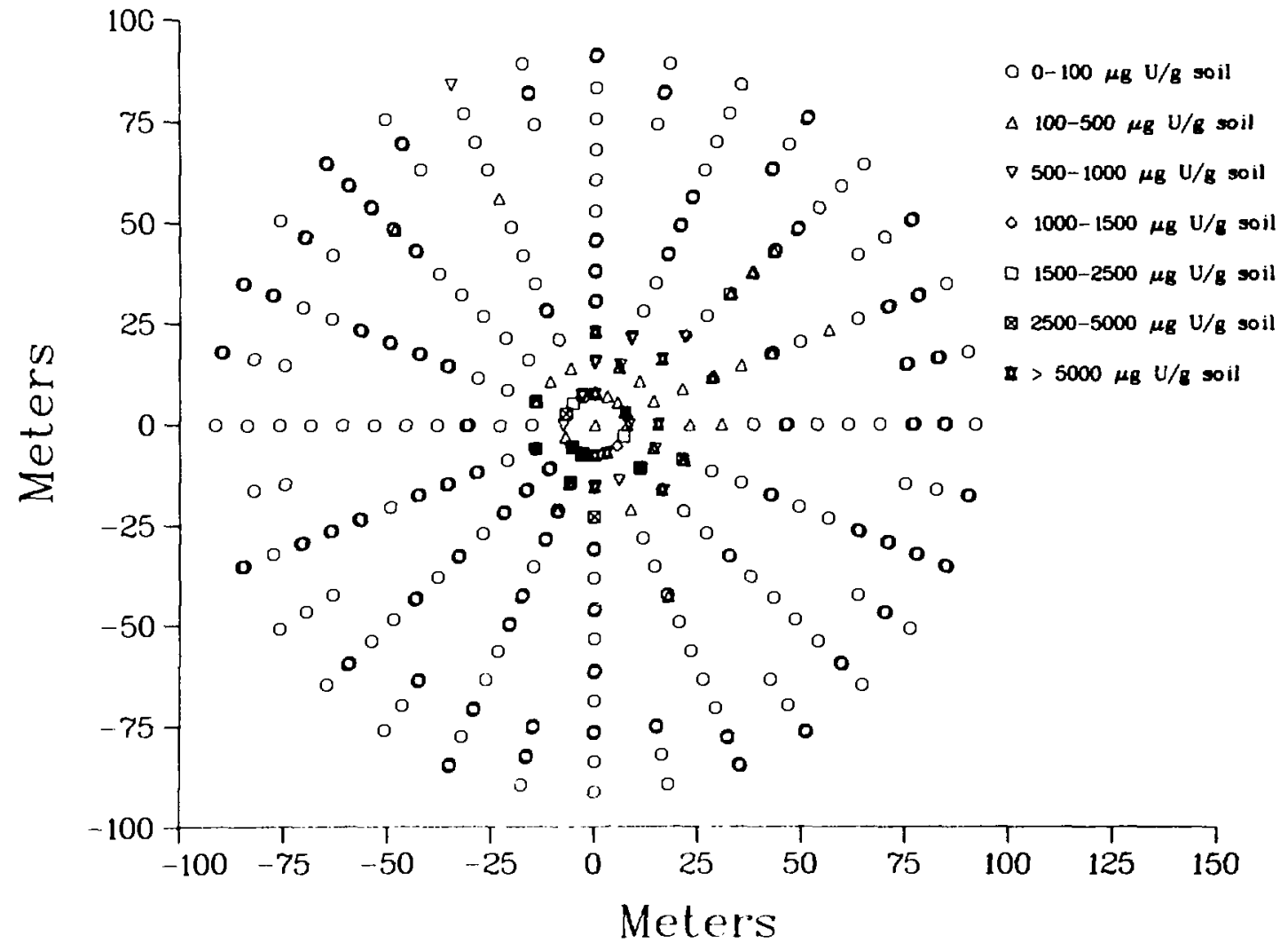

Fig. I.

The modified polar coordinate soil sampling design used at TA C. 74L.

$m$ larger than the previous one. Samples were taken at the intersections of these circles and 16 equally spaced rays from the center of the coordinate system, giving 192 samples. In addition, the three outer circles were sampled at the intersections of 16 more rays, equally spaced between the original 16, giving 48 more samples. To estimate the variability among samples taken close to each other, 100 of the previously described points were selected randomly. At each of these randomly chosen locations, additional samples were taken 0.2 and $0.6 \mathrm{~m}$ closer to the center along the rays. Thus, a total of 440 samples was taken.

This pattern retains a high sampling density near the target abutment, while increasing the sampling density in the outer areas. Also, the additional samples taken 0.2 and $0.6 \mathrm{~m}$ closer to other samples increased kriging's ability to quantify the nugget effect. The increased sampling intensity in the outer areas also helped to decrease the effect of localized uranium concentrations in the sampling.

\section{SAMPLE PREPARATION AND CHEMICAL ANALYSIS}

Each soil sample consisted of an area $10 \times 10 \times 0.5$ $\mathrm{cm}$ deep. Samples were collected by Eglin Air Force Base personnel during December 1979, then were shipped to Los Alamos and were ground in a Bico' ${ }^{\text {Rt }}$ Model UD pulverizer; a $\mathrm{I}-\mathrm{g}$ aliquot of each soil sample was taken for analysis. Soil samples were not sieved to remove the larger uranium chunks before grinding.

Instrumental epithermal neutron activation (IENA) analysis (Gladney et al. 1979) was performed on each I-g aliquot. In the IENA analysis, samples first were irradiated for $2 \mathrm{~min}$ with epithermal neutrons in the 
energy range 280 to $1000 \mathrm{eV}$. After a decay time of 2 to 4 days, each sample was counted for 5 min on a large $\mathrm{Ge}(\mathrm{Li})$ detector. The 228- and 278-keV transitions from the decay of ${ }^{239} \mathrm{~Np}$ (half-life of 2.35 days) were observed and were used for quantitative analysis. The gamma-ray spectra were accumulated on a pulse-height analyzer, and output was collected on computer-compatible magnetic tape for off-line data reduction. Soil samples from the Canadian Certified Reference Materials Project were used to verify the accuracy of the detection procedure.

\section{STATISTICAL ANALYSIS AND RESULTS}

Kriging, used to analyze the uranium concentrations in the surface soil at TA C-74L, provides an objective method of interpolating irregularly spaced sampling points to obtain contour maps and estimates of total uranium in the soil. This procedure uses the correlation between observations as a function of their separation, represented by a function known as a variogram. A brief description of kriging is given in White el al. (1980), with a more detailed description in Doctor (1979).

The program used for the kriging analysis, HSSR-CONTOUR (Campbell 1979), interpolates and contours spatial data. Some program modifications were made to accommodate the spatial scale of the TA C-74L uranium data.

The mean uranium concentration in the 440 samples was $274.6 \mu \mathrm{g} \mathrm{U} / \mathrm{g}$ soil with a standard error of 40.1 (the range was 0.6-8500). A histogram of the untransformed data and a histogram of the log-transformed data are presented in Fig. 2. Although the log-transform removed much skewness from the data, the log-transformed data still are not symmetrical.

An idea of the spatial variability of the data is given in Fig. 1, where different symbols represent uranium concentrations.

A contour map constructed from the kriging estimates is shown in Fig. 3. The majority of TA C-74L has uranium concentrations below $100 \mu \mathrm{g} \mathrm{U/g}$ soil. However, the area in and around : he target abutment has higher concentrations, reaching the observed high of $8500 \mu \mathrm{g} \mathrm{U} / \mathrm{g}$ soil south of the $(0,0)$ sampling point.

A contour map of the $95 \%$ confidence intervals, shown in Fig. 4, definitely reflects the sampling intensity. For example, the areas within the sampling grid with a uranium concentration confidence interval $>1750 \mu \mathrm{g}$
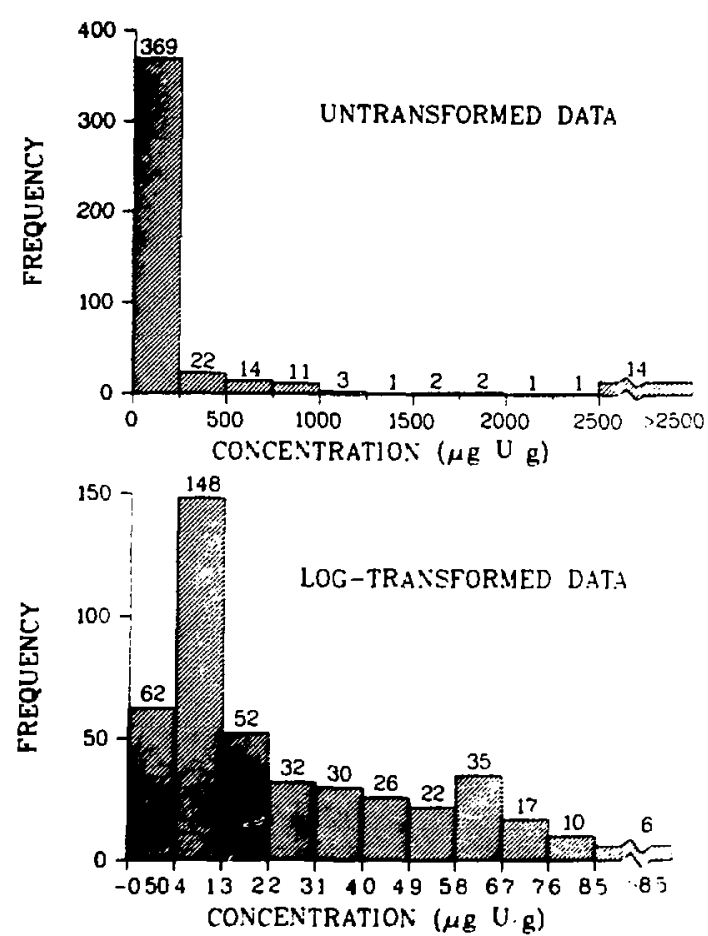

Fig. 2.

Histograms of untransformed and log-transformed uranium concentration data from TA C. $74 \mathrm{~L}$.

U/g soil are just inside the three outer rings where twice as many samples were taken. The locations of the sampling points can be detected easily as they radiate from the $(0,0)$ sampling point. The area around the target abutment has a smaller confidence interval than the area farther away, again reflecting the sampling intensity.

The actual portion of TA C-74L requiring clean-up appears small and restricted to the area center. Evidently, past activities in the area transported some contamination to the northeast of the target abutment, but relatively little movement of uranium has taken place.

The sampling design was not identical to that suggested by White el al. (1980) because a polar coordinate design already was available at TA C-74L, and it was logistically simpler to add additional rays and to increase the sampling intensity in the three outer rings. However, the results appear to be the same as would have been achieved by the $x-y$ coordinate system suggested by White et al. (1980). The greater intensity of samples at 


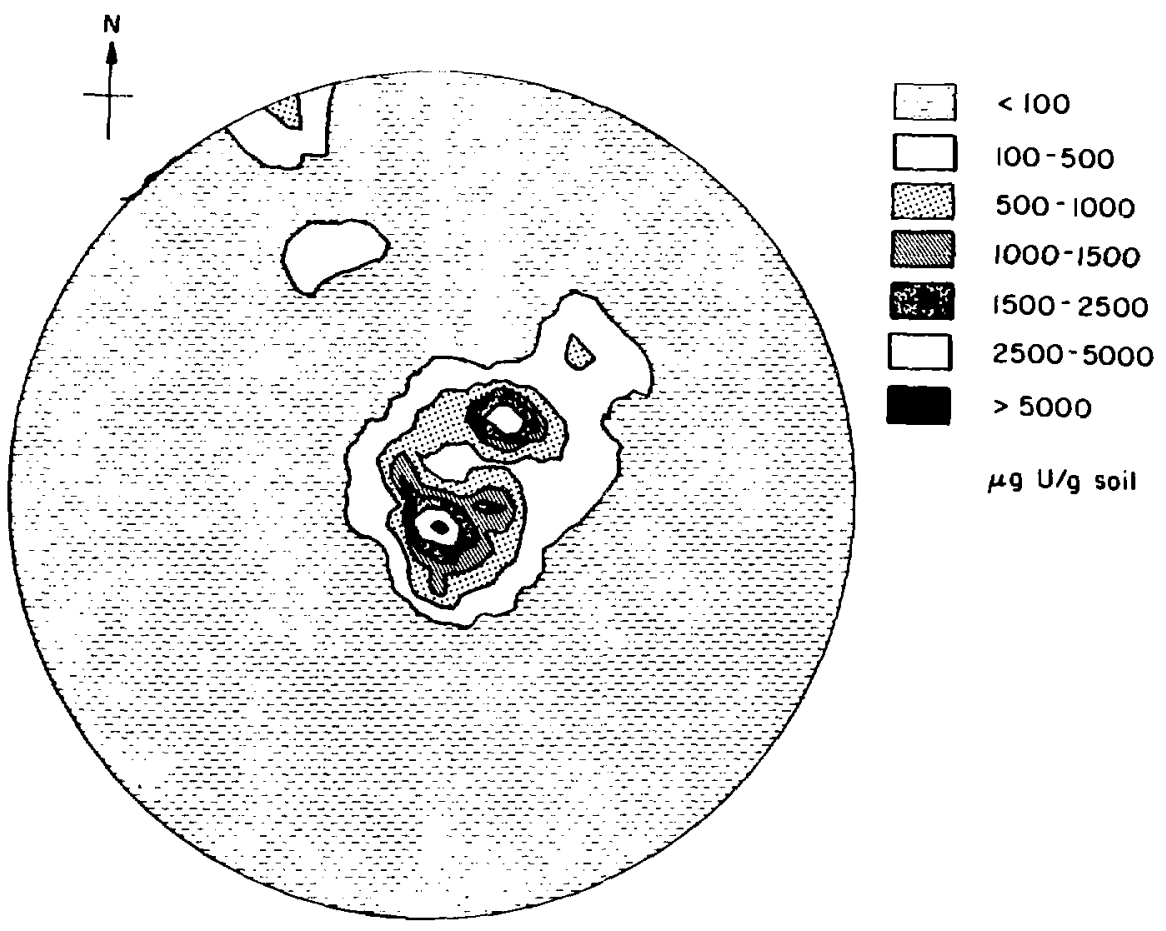

Fig. 3.

A contour map of uranium concentrations at TA C-74L. The outer circle has a $93-\mathrm{m}$ radius from the $(0,0)$ station.

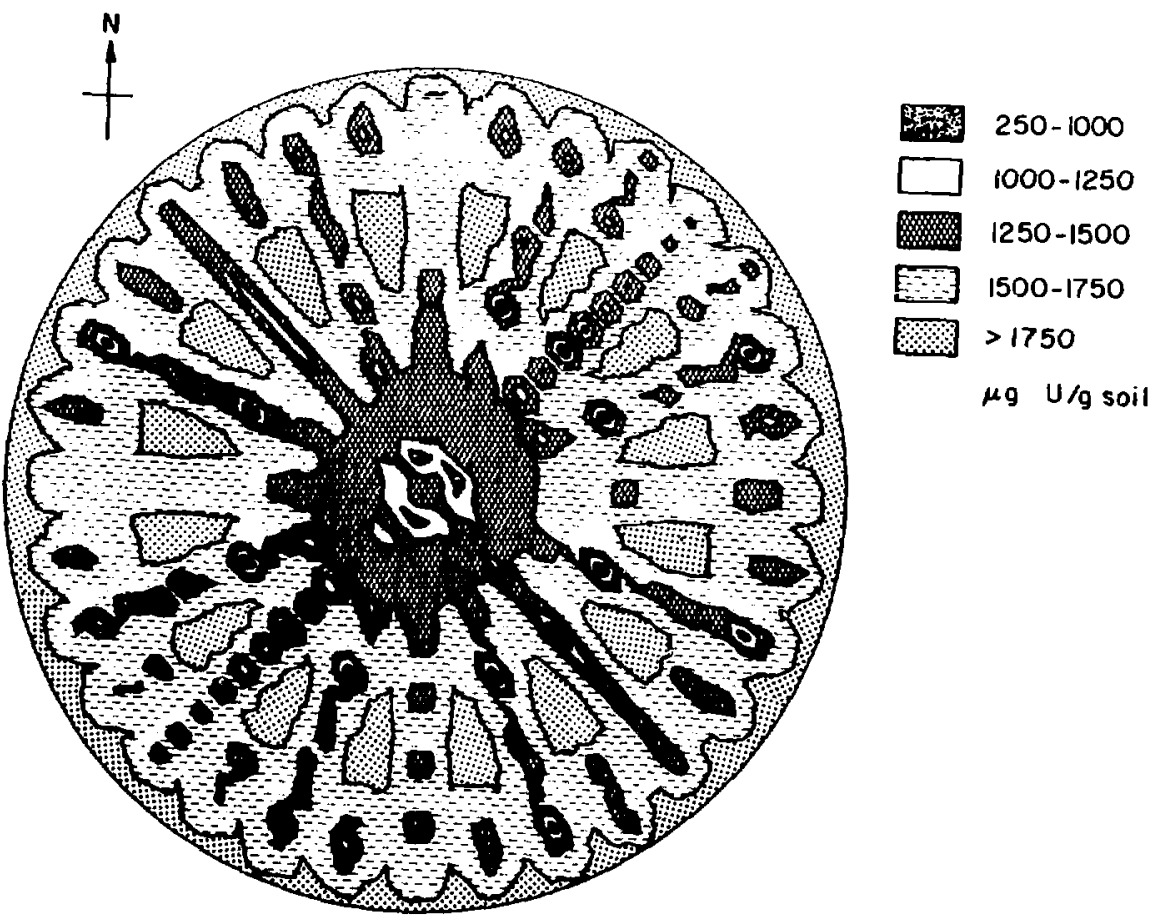

Fig. 4.

A contour map of the $95 \%$ confidence intervals. The outer circle has a $93-\mathrm{m}$ radius from the $(0,0)$ sation. 
the grid center still allows for better estimates in areas of highest concentrations, whereas the extra samples in the three outer rings provide a high enough sampling intensity to establish that very little uranium has moved from the target abutment.

\section{CONCLUSIONS}

Kriging again has provided a useful statistical tool to analyze concentrations of contaminants in soil. The contour map of concentrations and the associated contour map of $95 \%$ confidence intervals provide the necessary representation of the data to decide on clean-up procedures.

\section{ACKNOWLEDGMEN :}

I appreciate the efforts of E. M. Karlen, S. C. Best, K. V. Bostick, E. S. Gladney, and D. R. Perrin in preparing and chemically analyzing soil samples, and J. C. Cornette and $R$. Crews in obtaining the soil samples. $K$. Campbell allowed me to modify the kriging analysis programs developed at Los Alamos National Laboratory.

\section{REFERENCES}

Campbell, K., 1979, “User's Guide to HSSRCONTOUR: A Set of Programs for Interpolating and Contouring Hydrogeochemical Stream/Lake Sediment Reconnaissance Data from the NURE Program," Los Alamos National Laboratory, unpublished data.

Doctor, P. G., 1979, “An Evaluation of Kriging Techniques for High Level Radioactive Waste Repository Site Characterization," Battelle Pacific Northwest Laboratory report PNL-2903/UC-70.

Gladney, E. S., Owens, J. W., and Starner, J. W., 1979, "Simultaneous Determination of Uranium and Thorium in Ores by Instrumental Epithermal Neutron Activation Analysis," Anal. Chim. Acta 104, 121-127.

White, G. C., Simpson, J. C., and Bostick, K. V., 1980, "Studies of Long-Term Ecological Effects of Exposure to Uranium V," Los Alamos National Laboratory report LA-8221. 